\title{
Molecular contrast in optical coherence tomography using a pump-probe technique and a optical switch suppression technique
}

Changhuei Yang, K. Divakar Rao, Michael Choma, Siavash Yazdanfar, Andrew M. Rollins, et al.

Changhuei Yang, K. Divakar Rao, Michael Choma, Siavash Yazdanfar, Andrew M. Rollins, Joseph A. Izatt, "Molecular contrast in optical coherence tomography using a pump-probe technique and a optical switch suppression technique," Proc. SPIE 5140, Optical Coherence Tomography and Coherence Techniques, (2 October 2003); doi: 10.1117/12.501007

SPIE Event: European Conferences on Biomedical Optics 2003, 2003, Munich, Germany 


\title{
Molecular Contrast in Optical Coherence Tomography using a Pump-Probe Technique and a Optical Switch Suppression Technique
}

\author{
Changhuei Yang $^{1}$, K. Divakar Rao ${ }^{1}$, Michael Choma ${ }^{1}$, Siavash Yazdanfar ${ }^{1}$, \\ Andrew M. Rollins ${ }^{2}$, and Joseph A. Izatt ${ }^{1}$ \\ ${ }^{1}$ Department of Biomedical Engineering \\ Duke University, Durham, NC 27708 \\ ${ }^{2}$ Department of Biomedical Engineering \\ Case Western Reserve University, Cleveland, OH 44106
}

\begin{abstract}
We describe two novel technique for contrast enhancement in optical coherence tomography (OCT) which enables molecular specific imaging. The first, a pump-probe technique, is employed in which a pulsed pump laser is tuned to ground-state absorption in a molecule of interest. The location of the target molecule population is derived from the resulting transient absorption of OCT sample arm light acting as probe light. Preliminary results exhibiting contrast enhancement in cross-sectional OCT images using methylene blue dye are presented. The second method is an optical switch suppression technique based on the use of a transmembrane protein called bacteriorhodopsin. Initial experiments indicate that biochemical optical switches, such as bacteriorhodopsin, are excellent contrast agent candidates for molecular contrast OCT.
\end{abstract}

Keywords: OCT, molecular contrast, pump-probe spectroscopy

Optical coherence tomography (OCT) is an emerging tool for real time in-situ tissue imaging with micrometer-scale resolution. Real-time OCT systems have been integrated into clinical medical diagnostic instruments, and functional extensions such as polarization-sensitive, Doppler, and spectroscopic OCT have recently been introduced. These functional enhancements add the ability to discern contrast due to stress, motion, and to some extent absorber concentration in samples such as biological tissues, however OCT remains a relatively contraststarved imaging modality due to the low contrast in scattering coefficient between biological tissue types. OCT imaging technology has not yet taken advantage of molecular contrast mechanisms which have revolutionized other medical and biological imaging modalities such as MRI, PET, and SPECT. In particular, other optical techniques such as fluorescence, confocal, and multiphoton microscopy have had tremendous impact because of their capability to image highly specific molecular targets, including antibody-conjugated fluorescent probes and genetically expressible probes such as green fluorescent protein. Unfortunately, OCT is intrinsically insensitive to incoherent scattering processes such as fluorescence or spontaneous Raman scattering, because it depends upon coherent detection of scattered light. We have previously proposed a general concept for the use of spectroscopic OCT for detection of light from coherent inelastic scattering processes such as stimulated emission, stimulated Raman scattering, and other four-wave mixing processes [1]. In this report we present two new techniques. The first is based on a pump probe technique. It is a novel extension of OCT for enhancing the contrast of OCT images based on transient absorption in the sample induced by an 
external pump beam. The second is based on an optical switch suppression technique. The molecular contrast agents are biochemical optical switches, such as bacteriorhodopsin. These methods are centered on the exploitation of various optical properties in selected contrast agents to elicit changes in the OCT images acquired. These images can then be processed to reveal the concentration distribution of the contrast agents in the target.

The addition of molecular-specific contrast capability to OCT imaging would enable a large number of applications which are not presently possible with OCT. The potential applications for molecular contrast OCT technology include quantitative biological microscopy and noninvasive medical diagnostics. Laboratory OCT microscopy systems adapted for molecular contrast would enable quantitative imaging of exogenous probes and markers in cellular preparations and animal models, with improved imaging depth compared with fluorescence, confocal, or multi-photon microscopy. Contrast agents of interest for molecular contrast OCT include genetically expressible probes under the control of specialized promoters. Clinical OCT systems adapted for molecular contrast may enable diagnostic imaging of endogenous tissue chromophores relevant to disease processes, as well as in situ monitoring of therapeutic processes such as drug delivery.

\section{Pump-probe technique based molecular contrast OCT (PPOCT)}

Pump-probe spectroscopy is a standard tool in nonlinear optics for characterization of light induced changes in optical properties of materials, and for studying the relaxation dynamics of excited states [2]. We chose methylene blue as a demonstration contrast agent for PPOCT because it has spectrally distinct ground-state absorption in the visible region and triplet-triplet absorption in the $830 \mathrm{~nm}$ region, which is commonly used for OCT [3].

Generally speaking, pump probe OCT [4] relies on the use of a pump beam to transiently change the absorption of the target contrast agent at a probe wavelength which is used as the wavelength of an OCT imaging system. The difference in the OCT signal acquired at the probe wavelength as a function of the pump intensity can then be used to profile the contrast agent concentration distribution in the target sample. The transient nature of the target absorption can be used to implement time-gated or frequency-domain signal processing schemes which maximize the signal-to-noise ratio in the associated measurement. Such a scheme requires the use of contrast agents that have the requisite excitation energy level scheme that will permit a

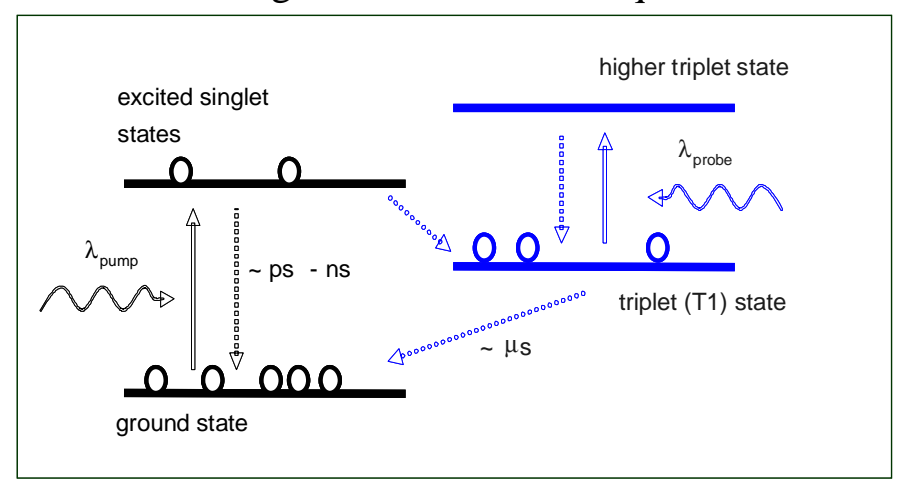

Figure 1: The energy level scheme for pump-probe excitation. For our experiment with methylene blue, the pump wavelength is $532 \mathrm{~nm}$ and the probe wavelength is $830 \mathrm{~nm}$. pump beam induced change of absorption coefficient at the probe wavelength. It is to be understood that the change in absorption may be of either sign, which is to say that the pump beam may either increase or decrease the absorption of the sample at the probe wavelength. It is also to be understood that the absorption itself may be of either sign; positive absorption corresponds to absorption in the conventional sense, and negative absorption corresponds to stimulated emission, which is a coherent process and is thus compatible with interferometric 


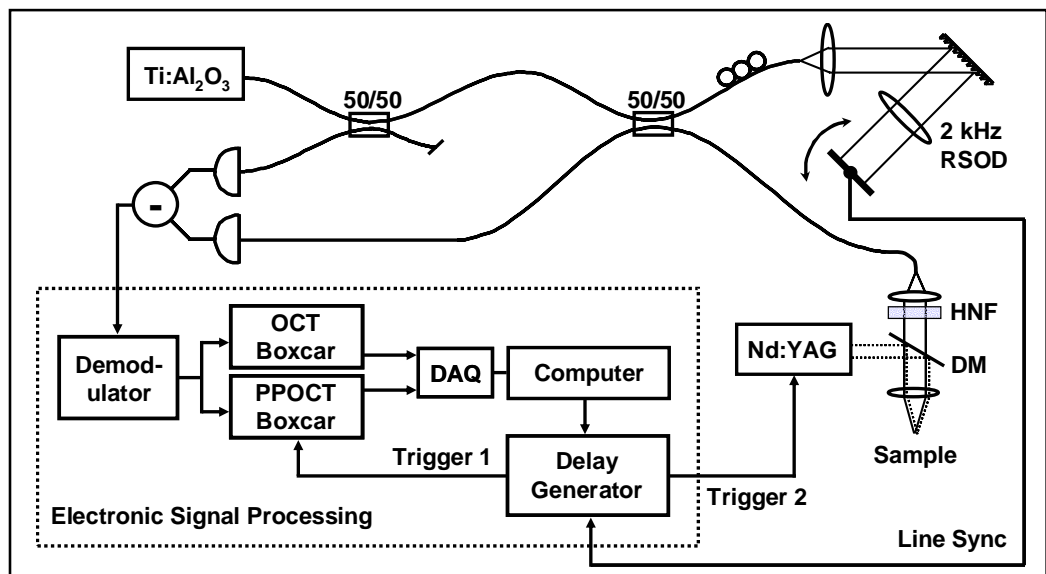

Figure 2: Experimental schematic for pump-probe optical coherence tomography. RSOD: rapid scanning optical delay line; DM: dichroic mirror; HNF: holographic notch filter; DAQ:data acquisition board.

pumped through a dichroic mirror by second harmonic light from a Q-switched Nd-Yag laser $(532 \mathrm{~nm}, \sim 50 \mathrm{~ns}$ pulse width, $10-50 \mu \mathrm{J} /$ pulse, $1 \mathrm{kHz}$ repetition rate, focal spot $\sim 40 \mu \mathrm{m})$. A

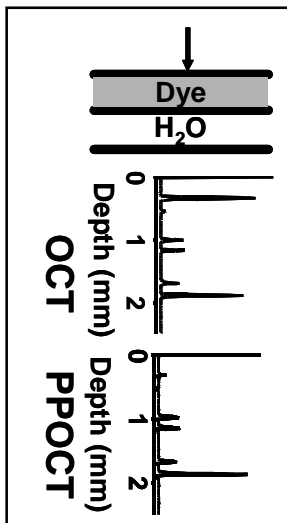

(a)

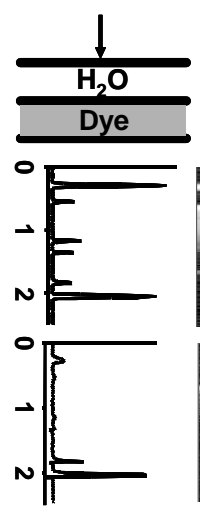

(b)

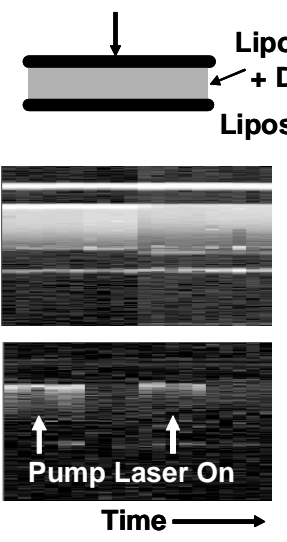

(c)

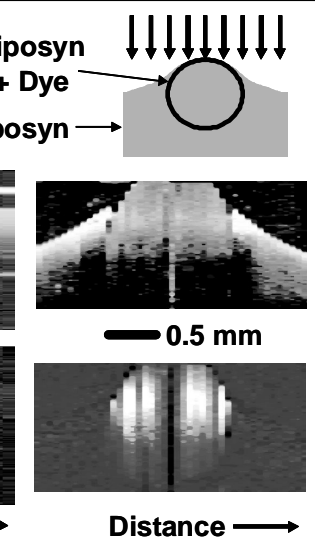

(d)
Figure 3: Columns (a) and (b), linear plots of 50 averaged OCT and PPOCT A-scans in a 2-level well phantom containing pure water and $\sim 0.5 \mathrm{mM}$ methylene blue dye in water in alternate locations. Differential PPOCT signals appear at phantom interfaces below the level of the dye. Column (c), log M-scans of OCT and PPOCT in a scattering medium $(\sim 0.5 \mathrm{mM}$ methylene blue dye in $0.5 \%$ Liposyn) with the pump laser alternatively blocked and unblocked. Column (d), $\log$ OCT and PPOCT crosssectional images of a capillary tube containing $\sim 0.5 \mathrm{mM}$ methylene blue dye in $0.5 \%$ Liposyn, partially immersed in $0.5 \%$ Liposyn without dye. PPOCT signal localized to within the tube is clearly visualized.
The experimental apparatus we used for PPOCT is illustrated in Fig. 2. A femtosecond Ti:sapphire laser output (center wavelength 800 $\mathrm{nm}$, spectral bandwidth $90 \mathrm{~nm}$ ), was used as the light source for OCT and a rapid scanning optical delay (RSOD) operating at $2 \mathrm{kHz}$ line rate was used for high speed acquisition of OCT A-scans. The source light was pulse-stretched with $\sim 30 \mathrm{~m}$ singlemode fiber to ensure that nonlinear excitation of the dye sample by the OCT probe light did not occur. The sample was holographic notch filter (HNF) prevented pump light from entering the OCT receiver. A computer-controlled delay generator triggered by the RSOD line sync triggered both the pump laser Q-switch and the synchronized detection electronics. Balanced detection was used to suppress non-interferometric transient absorption of sample arm light.

Demonstration of depthresolved molecular contrast imaging using pump-probe OCT in a nonscattering phantom is illustrated in Fig. 3 (a) and (b). The sample was a 2-level well phantom, consisting of three microscope cover slips spaced $\sim 500 \mu \mathrm{m}$ apart with pure water and $\sim 0.5$ $\mathrm{mM}$ methylene blue dye in water alternatively placed in

wells between the slips. OCT and PPOCT data were acquired with the dye in alternate 
compartments. Averaged OCT and PPOCT A-scans are displayed in columns (a) and (b), respectively. The depth-resolved PPOCT A-scans clearly show the presence of differential signal at the bottom of the dye layer and at all sequential interfaces, reflecting the cumulative nature of the PPOCT signal. Larger signals (both OCT and PPOCT) are observed at external (glass-air) as compared to internal (glass-water) interfaces. Small artifacts in the PPOCT scans are observed at the level of the top glass-air interface, possibly due to nonlinear receiver response at this large reflection.

Preliminary demonstrations of molecular contrast PPOCT imaging in a scattering medium were obtained using $\sim 0.5 \mathrm{mM}$ of methylene blue dye mixed into intravenous fat emulsion (Liposyn®, $\sim 0.5 \%$ solids). Figure 3 (c) illustrates $\log$ OCT and $\log$ PPOCT M-scans acquired within a single-layer cover slip phantom of $\sim .5 \mathrm{~mm}$ thickness, as the pump laser was alternately blocked and unblocked. A separate experiment (not shown) verified that no PPOCT signal was observed under identical experimental conditions, in the absence of dye in the scattering medium. Fig. 3 (d) illustrates log OCT and log PPOCT images of a glass capillary tube (outer diameter $\sim 1.6 \mathrm{~mm}$ ) containing $\sim 0.5 \mathrm{mM}$ of methylene blue dye in Liposyn, partially submerged in $0.5 \%$ lyposin without dye. The PPOCT image shows the signal only within the tube containing the dye. The central defect in the PPOCT image is attributed to saturation of the receiver from the strong reflection at the tube apex.

In summary, we have introduced a novel implementation of molecular contrast OCT that is capable of imaging molecular contrast distribution within a sample. Although the pump power levels and data acquisition times used in this preliminary study are not suitable for biological or medical applications, much more efficient implementations of PPOCT which optimize the temporal overlap of the pump and probe inputs are under development in our laboratory. The spectral selectivity of the technique offers flexibility to choose different contrast agents depending on the excitation source and transient spectra. The spatial and spectral selectivity of the nonlinear optical approach in PPOCT may have potential for enhancing OCT with the power of molecular-specific spectroscopic contrast for functional imaging.

\section{Biochemical optical 'switch' suppression molecular contrast OCT technique}

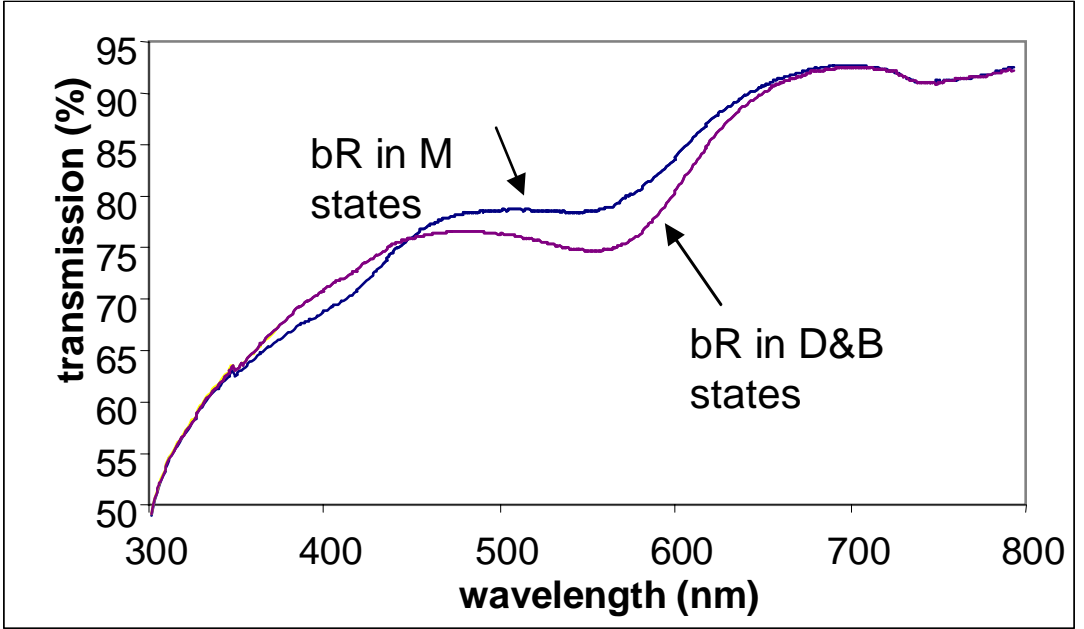

Figure. 4: Absorption spectra of $b R$ in $M$ and $D \& B$ states.
We have began experimentation with a new class of contrast agents biochemical species that can be reversibly switched from one stable state to another through a specific wavelength excitation. This state change also causes an associated change in the absorption spectrum. One notable example of such biochemical optical switch is bacteriorhodopsin (bR) [5]. This is a retinol based transmembrane protein that 
has evolved in swamp bacteria as part of the engine for converting sunlight into energy.

The advantages of using bR are numerous. First and foremost, it is, in principle, possible

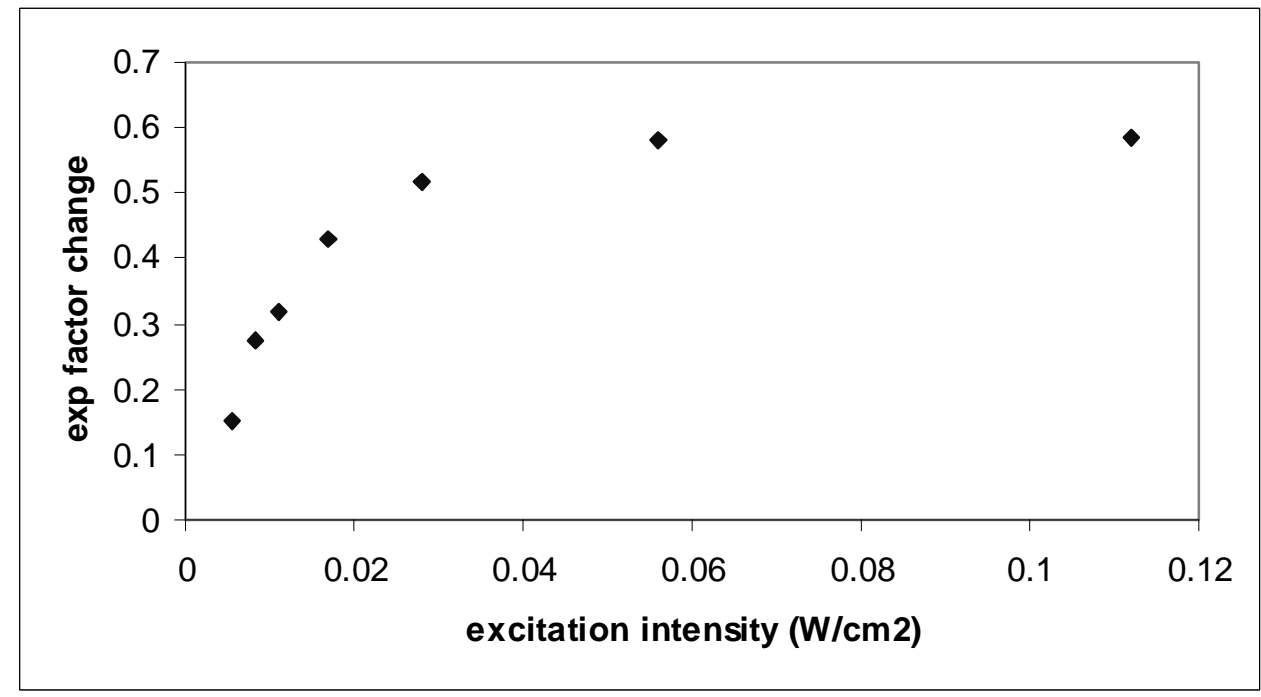

Figure 5: Experimental data showing that the optical density change at wavelength of $530 \mathrm{~nm}$ tapers off at about $75 \mathrm{~mW} / \mathrm{cm}^{2}$. to get other bacteria species or even mammalian cells to express the protein. This makes them very good potential MCOCT contrast agent candidate for filling the role that GFP is presently filling in fluorescence microscopy.

Second, the stability and absorption spectrum change between bR states

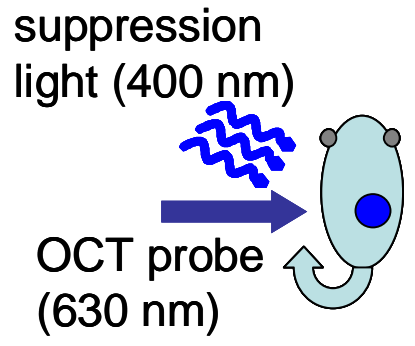
are extremely suited for $b R$ use as a MCOCT contrast agent.

Bacteriorhodopsin switches between two major state groups upon excitation. In its original D\&B state group, bacteriorhodopsin absorbs the red-green portion of the spectrum strongly. Upon exposure to light of wavelength 500-800 $\mathrm{nm}$, it transits into its $M$ state. In this state, bR absorbs strongly in the UV region of the spectrum. An experimental OCT images with the UV suppression light source

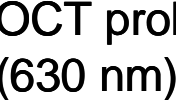

spectrum measurement scan of these 2
state groups of bR is shown in Fig. 4 (note that the transition was incomplete and the spectrum change less dramatic, due to the lack of available high light source intensity in the spectrophotometer). bR in $M$ state will transit back to D\&B state group upon excitation with UV light or after tens of milliseconds. The excitation intensity required for either state change is

Fig. 7: A-scans through $b R$ shows significant differences when UV beam suppression is on and off. 
dramatically low - measured to be $\sim 75 \mathrm{~mW} / \mathrm{cm}^{2}$ (equivalent to the intensity of a typical laser pointer), which makes state change in bR very easy to achieved. Fig. 5 shows that the optical density change in bR plateau at the intensity reported above.

With a probe wavelength of $630 \mathrm{~nm}$, we will actually cause bR within the sample to immediately make a transition to its $\mathrm{M}$ state at our typical OCT probe intensity. In order to attain OCT scans of bR distribution in its D\&B state, we use a state change suppression UV beam at $408 \mathrm{~nm}$. The experiment scheme for extracting molecular contrast information is illustrated in the cartoon in Fig. 6. This scheme has yielded great experimental feasibility promise. Figure 7 shows an A-scan through a $1 \mathrm{~mm}$ cuvette of $\mathrm{bR}$ acquired with the $100 \mu \mathrm{W}$ probe of $630 \mathrm{~nm}$ and a suppression $3 \mathrm{~mW} \mathrm{UV} \mathrm{beam} \mathrm{of} \mathrm{408nm.}$

\section{References}

1. J.A. Izatt, et al., U.S. Patent No. 6,002,480.

2. T. Fuji, T. Yoda, T. Hattori and H. Nakatsuka, Jpn. J. Appl. Phys. 39, 1738 (2000).

3. I. Carmichael and G. L. Hug, Journal of Physical and Chemical Reference Data 15, 1 (1986).

4. Rao, K.D., et al., Molecular contrast in optical coherence tomography by use of a pumpprobe technique. Optics Letters, 2003. 28(5): p. 340-342.

5. Haupst, U., J. Tittor, and D. Oesterhelt, Closing in on bacteriorhodopsin: progress in understanding the molecule. Annu. Rev. Biophys. Biomol. Struct., 1999. 28: p. 367. 